\title{
On correlated heterogeneities of glass-forming liquids
}

\author{
A. S. Bakai \\ National Science Center «Kharkov Institute of Physics and Technology», 61108 Kharkov, Ukraine \\ E-mail: bakai@kipt.kharkov.ua
}

Received June 3, 2002

\begin{abstract}
The thermodynamics and structure of glass-forming liquids are considered within the framework of the heterophase fluctuation (HPF) model. The main goal of the theory developed is to fined for a description of the long-range correlations (LRC) of the density fluctuations known as the Fischer cluster. The van der Waals approximation of the HPF model shows that the liquid can have an isolated solid-fluid critical point analogous to the critical point of a gas-liquid system. Heterophase fluctuations in the form of solidlike noncrystalline and fluidlike clusters can have LRC in a narrow vicinity of the critical point. An analysis shows that the properties of the conventional critical fluctuations differ from those of the Fisher cluster. This forces one to look for another explanation of the observed LRC in glass-forming liquids. Large configurational entropy of liquids and glasses is a manifestation of multiplicity of the short-range ordering of molecules in the amorphous solidlike and fluidlike clusters. The multiplicity of short-range order results in structural heterogeneities. Random-field Ginzburg - Landau equations for the HPFs are deduced taking into account the structural heterogeneities. The random field is generated by these heterogeneities. It is found that at least three characteristic correlation scales are inherent to the HPF: the radius of local order, $r_{0}$, which is comparable with the radius of the first coordination sphere; the random-field-controlled radius of critical fluctuations, $R_{c}$; the average correlation length of fractal aggregations formed by the correlated domains (the domains have size $\left.\sim R_{c}\right), \xi_{\mathrm{av}}$. The length $\xi_{\mathrm{av}}$ is the characteristic size of the Fischer cluster. The conditions for the appearance of the listed correlations are deduced by requiring that they provide minimization of the free energy of the system. The annealing kinetics and dynamics (the ultraslow modes) of the Fischer cluster are described.
\end{abstract}

PACS: 64.70.Pf

\section{Introduction}

Structural and dynamic heterogeneities of the glass-forming liquids have wide spectrum of space and time scales, see, e.g., [1] and the references cited therein. The most mysterious is the phenomenon of long-range correlations (LRC) of the density fluctuations (Fischer cluster) discovered and investigated during the last decade [2-9]. In a wide temperature range above the glass transition temperature $T_{g}$, the correlation length of density fluctuations is much larger than the molecule size. The annealing time of the Fischer cluster is many orders of magnitude larger than the $\alpha$-relaxation time $\tau_{\alpha}$. The main peculiarities of the Fischer cluster are following:
1) It exists in a rather large temperature range, $\sim 100 \mathrm{~K}$, above $T_{g}$.

2) The correlation length is up to $300 \mathrm{~nm}$, while the short-range order (SRO) radius $r_{0} \sim 1 \mathrm{~nm}$.

3) No critical behavior of the heat capacity, compressibility, and thermal expansion coefficient is observed above $T_{g}$.

4) The density fluctuations have a fractal structure of dimension $D<3$. The dimension depends on the temperature $T$ and the substance. It differs from the universal critical exponent of the pair correlation function.

5) Ultraslow modes characterizing the Fischer cluster dynamics are some orders of magnitude faster than the Fischer cluster equilibration (annealing) time. 
The $\alpha$-relaxation is insensitive to the Fischer cluster formation. The ultraslow dynamics is considerably slower than the $\alpha$-relaxation processes.

A few papers are devoted to theory of the Fischer cluster [10-12]. The approach proposed in [10] to description of the fluctuation patterning is not completed as yet. In [11] the LRC are treated as long-range critical fluctuations. They are described within the framework of a two-order-parameter model. But the features 3)-5) still have no proper explanation in [11].

In [12] it is shown that the LRC appears as result of aggregation of heterophase correlated domains (CDs) having the same short-range order. This paper is devoted to a further development of the theory.

We start from the idea that the glass-forming liquids have heterophase mesoscopic structure consisting of solidlike and fluidlike species. The average lifetime of each species is comparable with the $\alpha$-relaxation time $\tau_{\alpha}$. As a result of the rearrangements the species change their SRO and type (solidlike species become fluidlike and vice versa) but the average concentration of the species possessing a specified SRO is constant at the fixed pressure $P$ and temperature $T$. The fraction of molecules belonging to the solidlike species, $n_{s}(x, t)$, is chosen as the order parameter. It is a random field due to the randomness of the configurations formed by the species. The correlation properties of these configurations have to be found by minimization of the free energy of the system. These ideals form the basis of the heterophase fluctuation model of the glass-forming liquids. For brevity a liquid with developed heterophase fluctuations (HPFs) will be called a heterophase liquid.

In one form or another the idea of an important role of HPFs in the liquid-to-glass transition has been used in many treatments of the glass-forming liquids. In [13-23] the free energy of the heterophase liquid is described in the mean field approximation. Equations proposed in [12,14,15,17,22,23] show that the heterophase liquid can have a critical point. Moreover these equations are isomorphic to van der Waals theory of the gas-liquid critical point (see below). By adding a gradient term to the van der Waals free energy one can get the Ginzburg-Landau (GL) description of the gas-liquid states [24]. The same procedure allows one to use the GL equation for heterophase liquids [25]. Assuming that the supercooled liquid above $T_{g}$ is close to the critical point, one can get a description of the correlation phenomena in heterophase liquids. Apparently the formation of Fischer cluster possessing the properties 3)-5) cannot be treated as conventional critical fluctuations. This way of explanation of LRC fails. To come up with a solution of the problem staying in the framework of the HPF model, one has to take into account the SRO statistics and correlations. The free energy of a heterophase liquid is a functional of the order parameter and of the space distributions of the species possessing different SRO. In seeking the free energy one is led straightforwardly to the random-field Ginzburg-Landau model (RFGLM) [12]. Subsequent minimization of the free energy shows when and why the aforementioned correlations are beneficial.

It should be noted that the mean field approach is valid only outside the fluctuational critical the region [24] and that the location and size of fluctuational region depend strongly on the random field properties. Therefore the applicability conditions of the mean field model have to be checked.

The outline of this paper is as follows. Theoretical models of heterophase liquid states are considered in Sec. 2. The free energies of solidlike and fluidlike clusters are formulated in terms of constituent species possessing different free energies. The space distribution of these species in the framework of mean field theory (MFT) are described by fields $\mu_{s}(P, T, x), \mu_{f}(P, T, x)$; the subscripts «s» and «f» mean «solidlike» and «fluidlike», respectively. With the space-averaged quantities $\bar{\mu}_{S}(P, T)$ and $\bar{\mu}_{f}(P, T)$ the free energy takes the form of a two-species model which, as is shown below, is isomorphic to the van der Waals theory of a gas - liquid system. A generalization of the van der Waals theory including the gradient term and random fields $\mu_{s}(P, T, x)$ and $\mu_{f}(P, T, x)$ is a Ginzburg - Landau random-field model. Equations of this model are deduced. Short-range and long-range correlations of the random fields are investigated in Sec. 3. Theory of the critical point in the framework of RFGLM is developed in Sec. 4. Section 5 is devoted to the static properties of the long-range density fluctuations, while the dynamics of the heterophase fluctuations including description of the ultra-slow modes and annealing kinetics of Fischer's cluster are considered in Sec. 6. The conclusion in Sec. 7 includes a discussion of the theory developed.

\section{Theoretical models of the heterophase liquids}

The phenomenological description of HPLs usually starts with the introduction of the basic thermodynamic quantities $\mu_{f}, \mu_{s}$ and $\mu_{\text {int }}$, which are chemical potentials of the «pure» fluid and solid 
phases and of the interface, respectively. This last one for simplicity is assumed to be independent of the interface geometry. Here we are interested in consideration of the liquid states with comparable fractions of the solidlike and fluidlike clusters,

$$
0.15<n_{s}, n_{f}<0.85,
$$

where $n_{f}=1-n_{s}$ is the fraction of fluidlike clusters. The numbers 0.15 and 0.85 are the percolation thresholds of the clusters [26]. The condition (1) means that the solidlike and fluidlike species form interpercolating clusters.

The interpercolating heterophase states form easily when the interfacial free energy is small,

$$
0<\Delta \mu_{\text {int }}<<T
$$

where

$$
\Delta \mu_{\text {int }}=\mu_{\text {int }}-\frac{1}{2}\left(\mu_{f}+\mu_{s}\right) .
$$

The condition (2) provides for comparatively small (positive) contributions from the interfaces to the free energy of the system. As a result of that, the interface turns to be rather developed to gain large phase-mixing entropy. Because the phase formation is a cooperative phenomenon, the single molecules can not be statistically independent species in the heterophase states. The phase mixibility is characterized by the associativity parameters $k_{0}$. It determines the size of statistically independent units in calculations of the mixing entropy. Evidently, $k_{0}>1$.

\subsection{Chemical potentials of the pure phases}

Macroscopically homogeneous condensed amorphous phases - liquid and (or) noncrystalline solids - are heterogeneous on microscopic scales due to multiplicity of the SRO. The number of structure states of $N$ molecules $(N>>1)$ in these states can be presented in the following form (see, e.g., [27])

$$
W_{i}(N)=\exp \left(\zeta_{i} N\right), \quad i=s, f,
$$

$\zeta_{i}$ is the configurational entropy of ergodic states, or the complexity of nonergodic states,

$$
s_{c, i}=k_{B} \zeta_{i}
$$

and $k_{B}$ is Boltzmann's constant.

The multiplicity of the structure states reflects randomness of the potential relief of molecules. The set of minima of the relief is often called inherent structures $[27,28]$. The distribution of the potential energy minima, $\psi(\varepsilon, N)$, describing the density of structure states of a solid cluster in liquid, is [21]

$\psi_{s}(\varepsilon, N)=\frac{N}{\sqrt{2 \pi \delta_{s}^{2}}} \exp \left\{N\left[\zeta_{s}-\left(\varepsilon-\bar{\varepsilon}_{s}\right)^{2} / 2 \delta_{s}^{2}\right]\right\}$,

$\varepsilon$ is the potential energy per molecule at a minimum, $\bar{\varepsilon}_{s}$ is its average value, and $\delta_{s}^{2}$ is the variance per molecule.

The chemical potential of a noncrystalline solid cluster can be presented in the following form:

$$
\mu_{s}(P, T, x)=\bar{\mu}_{s}(P, T)+\tilde{\mu}_{s}(P, T, x),
$$

where $\bar{\mu}_{s}$ is the average value and $\tilde{\mu}_{s}$ describes variations of the chemical potential due to randomness of the potential relief. Ignoring dependence of the molecule vibrations and librations on the depth of potential well, we have $\tilde{\mu}_{s}(x) \approx \varepsilon(x)-\bar{\varepsilon}_{s}$.

The first and second moments of this random quantity are

$$
\begin{aligned}
& \left\langle\tilde{\mu}_{s}(x)\right\rangle=\frac{1}{V} \int \tilde{\mu}_{s}(x) d^{3} x=0, \\
& \left\langle\tilde{\mu}_{s}(x)\right\rangle^{2}=\frac{1}{V} \int \tilde{\mu}_{s}^{2}(x) d^{3} x=\delta_{s}^{2} .
\end{aligned}
$$

Here $V$ is volume of the system, and $\langle\ldots\rangle$ means the ensemble average, which is equal to space average due to ergodicity.

For $\bar{\mu}_{s}$ the following expression is obtained [21]:

$$
\bar{\mu}_{s}(P, T)=\bar{\varepsilon}_{s}-\frac{\delta_{s}^{2}}{T}-s_{C, s} T+\mu_{s, \mathrm{vib}}(P, T)+P v_{s} \text {. }
$$

Here $\mu_{s, \mathrm{vib}}$ is the vibrational part of the chemical potential, $v_{S}$ is the specific volume.

In analogy with (6)-(9) the chemical potential of the fluid phase can be written as

$$
\begin{gathered}
\mu_{f}(P, T, x)=\bar{\mu}_{f}(P, T)+\tilde{\mu}_{f}(P, T, x), \\
\bar{\mu}_{f}(P, T)=\bar{\varepsilon}_{f}-\frac{\delta_{f}^{2}}{T}-s_{c, f} T+ \\
+\mu_{f, \text { vib,trans }}(P, T)+P v_{f} .
\end{gathered}
$$

In the last term the translational mode of the motion is included.

The moments of $\tilde{\mu}_{f}(x)$, like (8), are

$$
\left\langle\tilde{\mu}_{f}(x)\right\rangle=0,\left\langle\tilde{\mu}_{f}^{2}(x)\right\rangle=\frac{1}{V} \int \tilde{\mu}_{f}^{2}(x) d^{3} x=\delta_{f}^{2} .
$$




\subsection{Mean field model of HPFs (van der Waals approximation)}

The average chemical potentials of the phases, $\bar{\mu}_{s}(P, T)$ and $\bar{\mu}_{f}(P, T)$, determined by (9) and (11), respectively, are used in this approximation. The chemical potential of the heterophase liquid is [22]

$$
\begin{aligned}
& \mu(P, T)=\bar{n}_{s} \bar{\mu}_{s}(P, T)+\left(1-\bar{n}_{s}\right) \bar{\mu}_{f}(P, T)+ \\
& +\bar{n}_{s}\left(1-\bar{n}_{s}\right) \Delta \mu_{\mathrm{int}}(P, T)+ \\
& +k_{0}^{-1} T\left[\bar{n}_{s} \ln \bar{n}_{S}+\left(1-\bar{n}_{s}\right) \ln \left(1-\bar{n}_{S}\right)\right],
\end{aligned}
$$

where $\bar{n}_{S}$ is the average value of the order parameter.

The solid - fluid coexistence curve on the $(P, T)$ plane is determined by the equation

$$
\bar{\mu}_{s}(P, T)=\bar{\mu}_{f}(P, T) .
$$

The equilibrium value of the order parameter $\bar{n}_{s}$ is determined by the equations

$$
\begin{gathered}
\frac{\partial \mu(P, T)}{\partial \bar{n}_{s}}=0, \\
\frac{\partial^{2} \mu(P, T)}{\partial \bar{n}_{s}^{2}}>0 .
\end{gathered}
$$

It follows from (13)-(16) that on the coexistence curve

$$
\bar{n}_{S}(P, T)=1 / 2 .
$$

The solution of this equation, which is a direct ana$\log$ of the critical isochore in the van der Waals theory of the gas-liquid critical point, gives the pressure dependence of the coexistence temperature $T_{e}(P)$, at which both phases are in equilibrium.

Routine analysis [22] shows that (15) has only one stable solution with

$$
0<\left.\Delta \mu_{\text {int }}\right|_{T_{e}}<\frac{2 T_{e}(P)}{k_{0}} .
$$

If

$$
\left.\Delta \mu_{\mathrm{int}}\right|_{T_{e}}>\frac{2 T_{e}(P)}{k_{0}},
$$

the equation (15) has three solutions in the vicinity of

$$
T_{c}=T_{e}\left(P_{c}\right)=k_{0} \Delta \mu_{\mathrm{int}} / 2 .
$$

Equation (20) determines the location of an isolated critical point on the coexistence curve. At this point a second-order phase transition takes place. On the coexistence curve below the critical point a first-order phase transition takes place. A continuous phase transformation with crossing of the coexistence curve occurs above the critical point. The condition $\Delta \mu_{\text {int }}>0$ in (17) is needed to provide the phase separation in the supercritical region.

It is worth noting once again that the expression (13) is isomorphic to the free energy of liquid - gas system in van der Waals theory (see [24]), but the order parameter used here is different. This follows immediately from (26) after omitting the gradient term and putting $h=\bar{\mu}_{s}-\bar{\mu}_{f}$.

An approximate solution of (13), (15) with the condition (18), is

$$
\bar{n}_{s} \approx \frac{1}{1+\exp (H \beta)},
$$

where

$$
H=\frac{k_{0}\left[\bar{\mu}_{s}(T)-\bar{\mu}_{f}(T)\right]}{1-k_{0} \Delta \mu_{\mathrm{int}} / 2 T_{e}(P)} .
$$

In the vicinity of the coexistence curve

$$
\begin{aligned}
& \mu_{s}(T)-\mu_{f}(T) \approx \Delta s\left(T_{e}\right)\left(T-T_{e}\right), \\
& \Delta s\left(T_{e}\right)=s_{f}\left(T_{e}\right)-s_{s}\left(T_{e}\right) .
\end{aligned}
$$

Here $s_{s}(T), s_{f}(T)$ is the entropy per molecule in the solid and fluid states, respectively.

Equation (21) describes the evolution of the order parameter with a continuous phase transformation.

It should be pointed out that the two-state, two-species, two-phase models considered in [13-17] formally are particular cases of (or can be presented in the form of) the equations (13)-(16).

As is shown in [23], the mean field approach developed gives a reasonable description of the observed continuous phase transformation in glassforming liquids. Along with that the above-mentioned LRC are outside the framework of this model.

\subsection{Random-field Ginzburg-Landau model}

The GL approach allows one to investigate the structure of HPFs in the vicinity of critical points. Near the coexistence curve the free energy with the fields $\nabla n_{S}(P, T, x)$ and $\tilde{\mu}_{s}(P, T, x), \tilde{\mu}_{f}(P, T, x)$ taken into account can be presented in the GL form [27]:

$$
\begin{aligned}
G & =G_{0}+G_{1}, \\
G_{0} & =N \bar{\mu}\left(P, T_{e}\right),
\end{aligned}
$$




$$
G_{1}=\frac{1}{v} \int\left[\frac{1}{2} A(\nabla \alpha)^{2}+\frac{1}{2} B \alpha^{2}+\frac{1}{4} C \alpha^{4}-h \alpha\right] d^{3} x,
$$

where $v$ is the specific volume,

$$
\begin{aligned}
& \alpha \equiv \alpha(P, T, x)=n_{s}(P, T, x)-\bar{n}_{s}\left(P, T_{e}\right)= \\
& =n_{s}(P, T, x)-\frac{1}{2} \\
& B \equiv 4 k_{0}^{-1} T-2 \Delta \bar{\mu}_{\mathrm{int}}(P, T)+\tilde{\mu}_{s}(x)+\tilde{\mu}_{f}(x) \equiv \\
& \equiv 4 k_{0}^{-1} T-2 \Delta \bar{\mu}_{\mathrm{int}}(P, T)+\tilde{\mu}_{\mathrm{int}}(x) \\
& \qquad=16 k_{0}^{-1} T / 3 \\
& h=\bar{\mu}_{s}(P, T)-\bar{\mu}_{f}(P, T)+\tilde{\mu}_{s}(P, T, x)- \\
& -\tilde{\mu}_{f}(P, T, x)=\bar{h}+\tilde{h}, \\
& \bar{h}=\bar{\mu}_{s}-\bar{\mu}_{f} ; \tilde{h}(x)=\tilde{\mu}_{s}(x)-\tilde{\mu}_{f}(x)
\end{aligned}
$$

The coefficient $A$ is connected with the interfacial energy,

$$
A=r_{0}^{2} A_{0} \sim r_{0}^{2} \Delta \mu_{\text {int }},
$$

and $r_{0}$ is the correlation radius outside the critical region; it is comparable with the molecular size. Apparently the associativity parameter $k_{0}$ is proportional to $r_{0}^{3}$,

$$
k_{0} \sim\left(r_{0} / a\right)^{3},
$$

where $a$ is characteristic size of molecule.

With $\tilde{h}=0, \nabla n_{s}(P, T, x)=0$, and $\tilde{\mu}_{\text {int }}=0$ the equations (24)-(26) are equivalent to (13) with accuracy $O\left(\alpha^{6}\right)$. In this case the free energy (26) is isomorphic to that of the van der Waals theory of the critical point.

The random field the $\tilde{h}(x)$ plays important role in formation of the order parameter and density fluctuations in the vicinity of $T_{c}$.

Equation (26) implies the saddle point equation:

$$
-A \Delta \alpha(x)+B \alpha(x)+C \alpha^{3}(x)=\bar{h}+\tilde{h}(x) .
$$

It describes both the conventional critical fluctuations and fluctuations driven by the random field $\tilde{h}(x)$. The equations (24)-(33) form the framework of the RFGLM.

\section{Correlation properties of the HPF}

\subsection{Critical fluctuations with $\tilde{h}(x)=0$}

To study out the role of random fields we start from a brief description of correlation phenomena without random fields, putting $\tilde{\mu}_{s}(x)=\tilde{\mu}_{f}(x)=0$. With $\tilde{h}(x)=0$ the equation (33) describes the conventional critical fluctuations of the order parameter in the absence of the random fields. The dimensionless parameter

$$
\tau_{0}=B_{e} / A_{0}, \quad B_{e}=4 k_{0}^{-1} T_{e}-2 \bar{\mu}_{\text {int }}\left(T_{e}\right)
$$

determines how close the system is to the critical point. In the region (18), this parameter is $\sim 2 / k_{0}$.

According to the conventional theory of critical fluctuations, the correlation radius of the order parameter fluctuations is

$$
\begin{gathered}
R_{c} \sim r_{0}\left|\tau_{0}\right|^{-v}, \bar{h} \ll \bar{h}_{c}(B), \\
R_{c} \sim r_{0}|\bar{h}|^{-\mu}, \bar{h}>>\bar{h}_{c}(B), \\
\bar{h}_{c}\left(B_{e}\right)=B_{e}\left(B_{e} / C\right)^{1 / 2},
\end{gathered}
$$

where $\nu, \mu$, and $\gamma$ are the critical exponents. They have the mean field values with

$$
\tau_{0} \gg\left(a / r_{0}\right)^{6} \sim k_{0}^{-2}
$$

when the mean field theory is valid ((36) is the Levanyuk-Ginzburg condition). In the fluctuational region, with $\tau_{0}<\left(a / r_{0}\right)^{6}$, the universal values of the critical exponents (known, e.g., from Wilson's theory) have to be used. It is worth noting that for $k_{0} \gg 1$ the fluctuational region is quite narrow.

The pair correlation function of the critical density fluctuations in the mean field approximation is known (see, e.g., [24]) to be

$$
\begin{gathered}
\bar{\rho}^{-2}\left\langle\delta \rho_{T}(x) \delta \rho_{T}\left(x_{1}\right)\right\rangle=\kappa_{T} T V^{-1} \delta\left(x-x_{1}\right), \\
\left|x-x_{1}\right| \gg>R_{c} .
\end{gathered}
$$

Here $\kappa_{T}$ is the compressibility of the HPL [22],

$$
\begin{aligned}
& \kappa_{T}=\frac{n_{s} v_{s} \kappa_{T_{s}}+\left(1-n_{s}\right) v_{f} \kappa_{f}}{n_{s} v_{s}+\left(1-n_{s}\right) v_{f}}+ \\
& +\frac{n_{s}\left(1-n_{s}\right)\left(v_{s}-v_{f}\right)^{2}}{\left[n_{s} v_{s}+\left(1-n_{s}\right) v_{f}\right]\left(B_{e}+3 C \alpha^{2}\right)},
\end{aligned}
$$

where $v_{s}$ and $v_{f}$ are the specific volumes of the solid and fluid fractions, respectively.

In the critical region $\kappa_{T} \sim\left|\tau_{0}\right|^{-\gamma} \sim R_{C}^{\gamma / v}, \gamma>0$ is a critical exponent. Accordingly to experimental 
data [4], the compressibility does not show any changes when the correlation length of Fischer cluster grows. Therefore the observed LRC can not be identified as the conventional correlation of critical fluctuations. If $\tilde{h}(x) \neq 0$, a new set of the correlation lengths can appear due to correlations of $\tilde{h}(x)$.

\subsection{Correlations driven by the random fields}

To consider the order parameter fluctuations generated by the random fields let us put

$$
\alpha(x)=\bar{\alpha}+\tilde{\alpha}(x) .
$$

Substitution of (39) into (33) and space averaging give the following equation:

$$
\begin{gathered}
\left(B_{e}+3 C \delta_{\alpha}^{2}\right) \bar{\alpha}+C \bar{\alpha}^{3}=\bar{h}, \\
\delta_{\alpha}^{2}=\frac{1}{V} \int \tilde{\alpha}^{2}(x) d^{3} x .
\end{gathered}
$$

Combining (33) and (39) and using averaging of the nonlinearities, it is easy to get

$$
-A \Delta \tilde{\alpha}(x)+\left(B+3 C \bar{\alpha}^{2}+3 C \delta_{\alpha}^{2}\right) \tilde{\alpha}(x)=\tilde{h}(x) .
$$

The resulting equation (41) includes two random fields, $\tilde{h}(x)$ and $\tilde{\mu}_{\text {int }}(x)$. It is known $[29,30]$ that the fluctuations $\tilde{\mu}_{\text {int }}(x)$ (fluctuations of $B$ ) result in strong coupling effects of the fluctuations in a small vicinity of the critical temperature, with $\left|T-T_{c}\right| \sim T_{c} \exp \left(-\right.$ const $\left./\left\langle\tilde{\mu}_{\text {int }}^{2}\right\rangle\right)$. This effect is small. We neglect it here and put $\tilde{\mu}_{\text {int }}=0$.

The equation (40) has solution (21) when $\delta_{\alpha}^{2}=0$.

To calculate $\delta_{\alpha}^{2}$ the equation (41) has to be solved. The solution is

$$
\begin{gathered}
\tilde{\alpha}(x)=\frac{1}{4 \pi A} \int K\left(\left|x-x_{1}\right|\right) \tilde{h}\left(x_{1}\right) d^{3} x_{1}, \\
K(r)=r^{-1} \exp (-\kappa r), \\
\kappa^{2}=\frac{\tilde{B}}{A}, \quad \tilde{B}=B_{e}+3 C \bar{\alpha}^{2}+3 C \delta_{\alpha}^{2} .
\end{gathered}
$$

Let us consider of the contribution of the random fields to the free energy. Combining (26) and (42), we have

$$
\tilde{G}=\tilde{G}_{1}+\tilde{G}_{\nabla},
$$

$$
\begin{aligned}
& \tilde{G}_{1}=-\frac{1}{2 v} \int \tilde{h}(x) \tilde{a}(x) d^{3} x= \\
& =-\frac{1}{8 \pi A v} \int \tilde{h}(x) K\left(\left|x-x_{1}\right|\right) \tilde{h}\left(x_{1}\right) d^{3} x d^{3} x_{1} \equiv \\
& \equiv-\frac{1}{8 \pi A v} \int K\left(\left|x-x_{1}\right|\right)\left\langle\tilde{h}(x) \tilde{h}\left(x_{1}\right)\right\rangle d^{3} x d^{3} x_{1},
\end{aligned}
$$

and

$$
\tilde{G}_{\nabla} \sim \frac{1}{2 v} \int A(\nabla \tilde{\alpha})^{2} d^{3} x
$$

is the contribution of the gradient term to the free energy. It is a positive quantity, while $\tilde{G}_{1}$ is negative. A minimum of $\tilde{G}$ can be achieved if $\tilde{G}_{\nabla}$ is minimal while $\tilde{G}_{1}$ has a maximum. It is seen that these conditions are independent of each other because $\tilde{G}_{\nabla}$ depends on the gradient of $\tilde{h}(x)$ on a scale $\sim R_{c}$, while $\tilde{G}_{1}$ is proportional to correlator of the random field $\tilde{h}(x)$. Therefore the short-range and even long-range correlations of $\tilde{h}(x)$ are needed to minimize $\tilde{G}_{1}$.

As follows from (42),

$$
(\nabla \tilde{\alpha}(x))^{2} \sim \kappa^{-2}(\tilde{h}(x))^{2}+\kappa^{-4}\left(\frac{\partial \tilde{h}(x)}{\partial x}\right)^{2}+\ldots
$$

The term proportional to $\partial \tilde{h} / \partial x$ is omitted in (47) because it gives zero after integration. It is seen from (46) and (47) that $\tilde{G}_{\nabla}$ has a minimal value, $\tilde{G}_{\nabla} \sim N A \kappa^{2}\left\langle\tilde{\alpha}^{2}\right\rangle$, if $\tilde{h}$ is nearly constant within CD and the second term of the right-hand side of (47) is small compared to the first.

Thus each CD is specified by the value of $\tilde{h}$ within it. This value is fluctuating around the mean $\langle\tilde{h}(x)\rangle_{c d}$, where $\langle\ldots\rangle_{c d}$ means averaging over CDs. The order parameter fluctuation within a CD has the estimate [24]

$$
\begin{gathered}
\left\langle\delta \tilde{\alpha}^{2}\right\rangle_{c d}=\frac{3 T v}{4 \pi A V_{c d}} \int_{0}^{V_{c}} K(|x|) d^{3} x \approx \frac{T}{4 z_{c d} \tilde{B}}, \\
z_{c d} \equiv \frac{V_{c d}}{v}=\frac{4 \pi R_{c}^{3}}{3 v}=\frac{4 \pi r_{0}^{3}}{3 v}\left(\frac{A_{0}}{\tilde{B}}\right)^{3 / 2} .
\end{gathered}
$$

Noting that (42) gives

$$
\tilde{\alpha} \approx \tilde{h} / \tilde{B},
$$

we see that therefore 


$$
\Delta_{h}^{2}=\left\langle\tilde{h}^{2}(x)-\left\langle\tilde{h}^{2}(x)\right\rangle_{c d}\right\rangle \approx \frac{T|\tilde{B}|}{4 z_{c d}} .
$$

Apparently a CD can be specified by a value $\tilde{h}$ just if

$$
\tilde{h}^{2}>\Delta_{h}^{2} .
$$

Because $\tilde{h}$ is a finite quantity, $|\tilde{h}| \leq \tilde{h}_{\max }<\infty$, the condition (51) has to be valid with $|\tilde{h}|=\tilde{h}_{\max }$, or otherwise the thermal fluctuations depress the random-field-driven effects.

The distribution of the random field values is

$$
P(\tilde{h})=\frac{1}{V} \int \delta[\tilde{h}(x)-\tilde{h}] d^{3} x,
$$

and

$$
c_{c d}(\tilde{h})=\frac{1}{V} \int_{\tilde{h}-\Delta_{h}}^{\tilde{h}+\Delta_{h}} \delta[\tilde{h}(x)-\tilde{h}] d^{3} x d \tilde{h} \approx 2 P(\tilde{h}) \Delta_{h}
$$

is the concentration of CDs with $|\tilde{h}(x)-\tilde{h}|<\Delta_{h}$.

In CDs with $|\tilde{h}|<\Delta_{h}$ the average value of field $\tilde{h}(x)$ is smaller than $\Delta_{h}$ and the field correlation effects are depressed by the fluctuations. The concentration of such domains, $c_{n c}$, is

$$
c_{n c} \approx 2 P(0) \Delta_{h} .
$$

The relations (52), (53) determine the distribution of CDs on $\tilde{h}$.

As it follows from (45) and (46) that the change of the free energy per molecule driven by field $\tilde{h}$ is

$$
g(\tilde{h}) \approx-\frac{\tilde{h}^{2}}{2 \tilde{B}} .
$$

Now it is clear that the LRC of the field $\tilde{h}(x)$ (when it exists) is a correlation of CDs having nearly the same values of the mean $\tilde{h}$. In other words, the CDs of nearly the same $\tilde{h}$ have to form a correlated aggregation to provide the long-range correlations of the field $\tilde{h}(x)$. The pair correlation function of such aggregation can be presented as

$$
\tilde{f}(\tilde{h}, r)= \begin{cases}1, & r<R_{c}, \\ r^{D(\tilde{h})-3} \exp (-r / \xi(\tilde{h})), & r>R_{c} .\end{cases}
$$

Here $D(\tilde{h}), \xi(\tilde{h})$ are the dimension and correlation length of the aggregation.

Because the average concentration of CDs within the aggregation is [31]

$$
c(D, \xi) \approx \frac{3}{4 \pi}\left(\frac{\xi}{R_{c}}\right)^{D-3}
$$

and $c(D, \xi(\tilde{h}))=c_{c d}(\tilde{h})$, the correlation length $\xi(\tilde{h})$ is related to the concentration $c_{c d}(\tilde{h})$ :

$$
\xi(\tilde{h})=R_{c}\left[c_{c d}(\tilde{h})\right]^{\frac{1}{D-3}}=R_{c}\left[2 P(\tilde{h}) \Delta_{h}\right]^{\frac{1}{D-3}} .
$$

The (fractal) dimension $D$ depends on the nature of the aggregating species. Usually it is within the range 1.8-2.5 [31]. There is no reason to suspect that it depends strongly on $\tilde{h}$. Therefore we consider it here as an $\tilde{h}$-independent constant which is a property of the liquid.

To show that the aggregation of CDs diminishes the free energy we compare the free energy of a liquid with aggregated and nonaggregated CDs. With nonaggregated CDs, due to (50), (51)

$$
\tilde{H}(r)=\left\langle\tilde{h}(x) \tilde{h}\left(x_{1}\right)\right\rangle \approx\left\langle\tilde{h}^{2}(x)\right\rangle=\delta_{h}^{2}
$$

and

$$
\tilde{G}_{1}=-\frac{N}{2 A} \int K(r) \tilde{H}(r) r^{2} d r \approx-\frac{N \delta_{h}^{2} R_{c}^{2}}{2 A_{0} r_{0}^{2}} .
$$

When the CDs are aggregated we have to use (56), and

$$
\tilde{H}(r) \approx\left\{\begin{array}{lr}
\delta_{h}^{2}, & r<R_{c}, \\
\int P(\tilde{h}) \tilde{h}^{2} \tilde{f}(\tilde{h}, r) d \tilde{h}, & r>R_{c}
\end{array}\right.
$$

or

$$
\tilde{H}(r) \approx \begin{cases}\delta_{h}^{2}, & r<R_{c}, \\ \delta_{h}^{2} r^{D-3} \exp \left(-r / \xi_{\mathrm{av}}\right), & r>R_{c} .\end{cases}
$$

Here $\xi_{\text {av }}$ is the average correlation length and $D$ is the average fractal dimension.

Substitution of (62) into (45) gives

$$
\tilde{G}_{1}(\xi) \approx-\frac{N \delta_{h}^{2} R_{c}^{2}}{8 A_{0} r_{0}^{2}}\left[1+4 e^{-1}\left(\frac{\xi_{\mathrm{av}}}{R_{c}+\xi_{\mathrm{av}}}\right)^{D-1}\right] .
$$

It is seen from (60), (63) that the fractal aggregation of CDs really does lower the free energy.

Because $\tilde{G}_{1} \sim-\delta_{h}^{2}$, the random fields $\tilde{\mu}_{s}(x), \tilde{\mu}_{f}(x)$ have to provide a maximal value of $\delta_{h}^{2}$ for minimization of the free energy $\tilde{G}_{1}$. Variation of 


$$
\delta_{h}^{2}=\frac{1}{V} \int \tilde{h}^{2}(x) d^{3} x=\frac{1}{V} \int\left[\tilde{\mu}_{s}(x)-\tilde{\mu}_{f}(x)\right]^{2} d^{3} x
$$

with conditions (41), (45) and the assumption that the distributions of $\tilde{\mu}_{s}, \tilde{\mu}_{f}$ can be approximated by Gaussian function with variances gives that (63) attains a maximum for

$$
\tilde{\mu}_{s}(x)=-\left(\delta_{s} / \delta_{f}\right) \tilde{\mu}_{f}(x) .
$$

This result is evident: in this case $\delta_{h}^{2}$ has the maximal value, $\delta_{h}^{2}=\left(\delta_{s}+\delta_{f}\right)^{2}$. If $\tilde{\mu}_{s}(x)$ and $\tilde{\mu}_{f}(x)$ are uncorrelated, then $\delta_{h}^{2}=\delta_{s}^{2}+\delta_{f}^{2} \leq\left(\delta_{s}+\delta_{f}\right)^{2}$.

Is worth noting that the relation (64) describes the correlation of solid and fluid short-range orders within CDs. Thus the inherent structures of the solid and fluid fractions are correlated on this scale.

The entropy of the system is reduced due to the correlations. The main entropy reduction is caused by the inhomogeneous redistribution of species having the same SRO in the granulated liquid. Due to relation $(65) \tilde{\mu}_{s}(x)$ and $\tilde{\mu}_{f}(x)$, i.e., the SRO of the solidlike and fluidlike species, are determined by $\tilde{h}(x)$. When the species of the same $\tilde{h}$ are collected in CDs of size $z_{c}$ the mixing entropy per molecule is

$$
s(\tilde{h})=2 z_{c}^{-1} \ln c_{c d}(\tilde{h}) .
$$

Note that when they are homogeneously distributed, one has $s(\tilde{h})=k_{0}^{-1} \ln c_{c d}(\tilde{h})$. Thus the entropy reduction due to formation of correlated domains is

$$
\Delta s(\tilde{h})=k_{0}^{-1} \ln c_{c d}(\tilde{h})\left(1-2 k_{0} / z_{c}\right) .
$$

It follows that the total change of free energy per molecule due to the field $\tilde{h}$ is

$$
\Delta g(\tilde{h})=-\frac{\tilde{h}^{2}}{2 \tilde{B}}+T \Delta s(\tilde{h}) .
$$

It has to be negative,

$$
\frac{\tilde{h}^{2}}{2 \tilde{B}}>T \Delta s(\tilde{h}),
$$

to provide the random-field-driven formation of CDs.

\section{Random-field-driven critical fluctuations}

The correlation properties of the random fields result in correlations of the order parameter and density fluctuations. Besides the location of the critical point, even the order of the phase transition can change due to the random field, as is shown below.

\subsection{Critical fluctuations and location of the critical point}

Returning to the Eqs. (40)-(42) and taking into account (50), (51), we have

$$
\tilde{\alpha}(x)=\frac{\tilde{h}(x)}{\tilde{B}}+O\left(\frac{1}{\kappa \xi}\right)
$$

and

$\left(B_{e}+3 C \tilde{\delta}_{\alpha}^{2}\right) \bar{\alpha}+C \bar{\alpha}^{3}=\left[B_{e}+\frac{3 C \delta_{h}^{2}}{\tilde{B}^{2}}\right] \bar{\alpha}+C \bar{\alpha}^{3}=\bar{h}$,

where it is considered that

$$
\delta_{\alpha}^{2}=\delta_{h}^{2} / \tilde{B}^{2} .
$$

These relations complete the RFGLM equations.

The impact of the chemical potential fluctuations on the location of the critical point is seen from (71), (72). On the coexistence curve $\left(T=T_{e}\right)$ these equations give

$$
\tilde{B}_{e} \bar{\alpha}+C_{e} \bar{\alpha}^{3}=0, \quad \tilde{B}_{e}=B_{e}+3 C_{e} \delta_{\alpha}^{2} .
$$

Equation (72) has only one solution, $\bar{\alpha}=0$, with $\tilde{B}_{e}>0$, and two additional solutions

$$
\bar{\alpha}_{1,2}= \pm\left(-\tilde{B}_{e} / C_{e}\right)^{1 / 2}
$$

with $\tilde{B}_{e}<0$.

With $\bar{\alpha}^{2}=0$, i.e., on the coexistence curve above the critical point, the equation (72) has just one solution,

$$
\delta_{\alpha, 1}^{2} \approx\left(\frac{\delta_{h}^{2}}{9 C_{e}^{2}}\right)^{1 / 3}-\frac{2 B_{e}}{9 C_{e}}
$$

if

$$
B_{e}>B_{e, c}=-9 C_{e}\left(\frac{\delta_{h}^{2}}{36 C_{e}^{2}}\right)^{1 / 3} .
$$

For $B<B_{e, c}$ and $\bar{\alpha}^{2}=0$ the equation (72) has three real solutions. The additional two roots turn out less than $\delta_{\alpha, 1}$. Because the free energy $\tilde{G}_{1}$ is proportional to $-\delta_{\alpha}^{2}$, it has a minimal value for $\delta_{\alpha}^{2}=\delta_{\alpha, 1}^{2}$. Therefore the aforementioned additional roots of (72) with $B_{e}<B_{e, c}$ belong to unstable and metastable states and are not of interest to us. 
A similar analysis with $\bar{\alpha} \neq 0$ shows that Eq. (72) has three real roots with

$$
B_{e}<B_{e, c 1}=-9 C_{e}\left(\frac{\delta_{h}^{2}}{144 C_{e}^{2}}\right)^{1 / 3} .
$$

The smallest of them, $\delta_{\alpha, 2}^{2}$, is

$$
\begin{aligned}
& \delta_{\alpha, 2}^{2} \approx \frac{\delta_{h}^{2}}{4 B_{e}}\left(1-\frac{3 C_{e} \delta_{h}^{2}}{2 B_{e}^{3}}\right), \\
& \left|B_{e}-B_{e, c 1}\right|>\left|B_{e, c 1}\right| ; \\
& \delta_{\alpha, 2}^{2} \cong\left(\frac{\delta_{h}^{2}}{24 C_{e}^{2}}\right)^{1 / 3}\left[1-2\left(\frac{B_{e, c 1}-B_{e}}{B_{e, c 1}}\right)^{1 / 2}\right], \\
& B_{e, c 1}-B_{e} \sim\left|B_{e, c 1}\right| .
\end{aligned}
$$

It provides a minimal value of the free energy (which is less than that with $\bar{\alpha}^{2}=0$ and $\delta_{\alpha}^{2}=\delta_{\alpha, 1}^{2}$ ) when

$$
B_{e}<B_{e}^{*} \approx-2\left(C_{e} \delta_{h}^{2}\right)^{1 / 3}
$$

Thus the average value of the order parameter, $\bar{\alpha}$, for $B_{e}=B_{e}^{*}$ is nonzero,

$$
\bar{\alpha}>\bar{\alpha}_{c} \approx\left(B_{e}^{*} / C_{e}\right)^{1 / 2},
$$

for $B_{e}<B_{e}^{*}$, but $\bar{\alpha}=0$ for $B_{e}>B_{e}^{*}$.

This result shows that at $B_{e}=B_{e}^{*}$, i.e., at

$$
\begin{aligned}
& T_{c}\left(\delta_{h}, P\right)=\frac{k_{0} \Delta \mu_{\mathrm{int}}\left(P, T_{e}\right)}{2}-\frac{k_{0}}{2}\left(C \delta_{h}^{2}\right)^{1 / 3}= \\
& =T_{c}(0)-\frac{k_{0}}{2}\left(C \delta_{h}^{2}\right)^{1 / 3},
\end{aligned}
$$

a first order phase transition takes place. $T_{c}(0)$ is the critical temperature of system for $\tilde{h}=0$. The order parameter jump at $T=T_{c}\left(\delta_{h}, P\right)$, accordingly to $(80)$, is $\sim\left(\delta_{h}\right)^{1 / 3}$. Thus the random field changes not only the critical point location but also the order of the phase transition.

A similar result is known for the mean field version of the random field Ising model (RFIM) with a frozen-in random field [29,30].

The relations obtained show that for

$$
\left|B_{e}\right|=A_{0} \tau_{0}<2\left(C_{e} \delta_{h}^{2}\right)^{1 / 3} \text { and }|\bar{h}|<\tilde{h}_{\max }
$$

the random-field-driven fluctuations of the order parameter are dominant. Taking into account the condition (36), the first of relations (82) can be presented as follows:

$$
2\left(C_{e} \delta_{h}^{2}\right)^{1 / 3}>>A_{0} k_{0}^{-2} .
$$

The relation (82) determines the critical region of the RFGLM, where $\tilde{B} \sim\left(C_{e} \delta_{h}^{2}\right)^{1 / 3}$ and

$$
R_{c}^{3} \sim r_{0}\left(A_{0} / \tilde{B}\right)^{3 / 2} \sim r_{0}^{3}\left(A_{0}^{3} / C \delta_{h}^{2}\right)^{1 / 2} .
$$

Condition (51) and relation (84) show that the effects considered correlation are relevant if

$$
\delta_{h}^{2}>\left(\frac{a}{r_{0}}\right)^{18} \frac{T^{6} C^{5}}{A_{0}^{9}} \sim \frac{T^{6} C^{5}}{k_{0}^{6} A_{0}^{9}} .
$$

Noting that the coefficients $A_{0}, C, T$ all are of the same order of magnitude, we see that the condition (85) is compatible with condition (83).

The condition (69) in the critical region (where $\left.\tilde{B} \sim\left(C_{e} \delta_{h}^{2}\right)^{1 / 3} \sim\left(T \delta_{h}^{2}\right)^{1 / 3}\right)$ reads

$$
\begin{aligned}
& \tilde{h}^{2}>\tilde{h}_{c}^{2}=\frac{2(1-2 x)}{k_{0} x^{4 / 3}} \delta_{h}^{2} \ln c_{c d}(\tilde{h}), \\
& x=\delta_{h} / T .
\end{aligned}
$$

Thus the RFGLM used here is valid when the variance of the random field obeys the condition (85) and LRC are setting in if there are some CDs for which the inequality (86) is fulfilled.

\section{Long-range density fluctuations}

It follows from (24)-(26) that the specific volume of liquid is

$$
v=\frac{1}{2}\left(v_{f}+v_{s}\right)-\left(v_{f}-v_{s}\right) \alpha(x) .
$$

Density fluctuations include the conventional thermal fluctuations, $\delta \rho_{T}(x)$, around $\bar{\rho}$ and the random-field-driven fluctuations $\tilde{\rho}(x)$. It follows from (42) and (87) that the random-field-driven density fluctuations have the following pair correlation function:

$$
\begin{aligned}
& \frac{1}{\bar{\rho}^{2}}\left\langle\tilde{\rho}(x) \tilde{\rho}\left(x_{1}\right)\right\rangle=\left(\frac{v_{s}-v_{f}}{\bar{v}}\right)^{2}\left\langle\tilde{\alpha}(x) \tilde{\alpha}\left(x_{1}\right)\right\rangle= \\
& =\left(\frac{v_{s}-v_{f}}{\bar{v}}\right)^{2} \frac{\tilde{H}\left(\left|x-x_{1}\right|\right)}{\tilde{B}^{2}} .
\end{aligned}
$$

Noting that the correlation function (88) has to be added to (37) to get the complete pair correlation function, we have 


$$
\begin{aligned}
& \frac{1}{\bar{\rho}^{2}}\left\langle\delta \rho(x) \delta \rho\left(x_{1}\right)\right\rangle= \\
& =\frac{1}{\bar{\rho}^{2}}\left[\left\langle\delta \rho_{T}(x) \delta \rho_{T}\left(x_{1}\right)\right\rangle+\left\langle\tilde{\rho}(x) \tilde{\rho}\left(x_{1}\right)\right\rangle\right] \cong \\
& \cong \kappa_{T} T \frac{1}{V} \delta\left(x-x_{1}\right)+ \\
& +\left(\frac{v_{s}-v_{f}}{\bar{v}}\right)^{2} \frac{\left(\delta_{s}+\delta_{f}\right)^{2}}{\tilde{B}^{2}} \frac{1}{r^{3-D}} \exp \left(-\frac{r}{\xi}\right) .
\end{aligned}
$$

The first term of the right-hand side of (89) is proportional to $\tilde{B}^{-1}$ (see (38)), while the second is $\sim \widetilde{B}^{-2}$. Therefore the random-field-driven density fluctuations are dominant when condition (85) is fulfilled.
Fourier transformation of (89) gives spectral components $\delta \rho_{q}^{2}$ :

$$
\begin{aligned}
& \frac{\rho_{q}^{2}}{\bar{\rho}^{2}}=\kappa_{T} T \frac{1}{V}+\left(\frac{v_{s}-v_{f}}{\bar{v}}\right)^{2} \frac{\left(\delta_{s}+\delta_{f}\right)^{2}}{\tilde{B}^{2}} \times \\
& \times \frac{\Gamma(D-1)}{\left(1+q^{2} \xi^{2}\right)(D-1) / 2} \times \\
& \times\left(\frac{\xi}{R_{c}}\right)^{D} \frac{\sin [(D-1) \arctan (q \xi)]}{q \xi},
\end{aligned}
$$

where $\Gamma(x)$ is the gamma function.

The second term of the right-hand side of (90) describes the random-field-driven heterophase fluctuations $\tilde{\rho}_{q}^{2}$. It has simpler forms in two limiting cases, with small $q$ and with $q \xi>1$ :

$$
\frac{\tilde{\rho}_{q}^{2}}{\bar{\rho}^{2}}= \begin{cases}\left(\frac{v_{s}-v_{f}}{\bar{v}}\right)^{2} \frac{\left(\delta_{s}+\delta_{f}\right)^{2} \Gamma(D)}{\tilde{B}^{2}}\left(\frac{\xi}{R_{c}}\right)^{D}, & q \rightarrow 0, \\ \left(\frac{v_{s}-v_{f}}{\bar{v}}\right)^{2} \frac{\left(\delta_{s}+\delta_{f}\right)^{2}}{\tilde{B}^{2}} \frac{\Gamma(D-1)}{(q \xi)}\left(\frac{\xi}{R_{c}}\right)^{D} \sin \left[(D-1) \frac{\pi}{2}\right], & q \xi>1 .\end{cases}
$$

\section{Time-dependent GL equations}

The HPFs have short-range correlation on a scale $r \sim R_{c}$ and long-range correlation with $r \sim \xi$. The parameters $\delta_{n}^{2}, \quad \xi$ and the Fourier components $\left\{\tilde{\alpha}_{q}\right\}$ are time-dependent quantities. Conventional dynamic equations for them describe the short-range and long-range relaxation as well as the ultraslow modes. We are using here the time-dependent GL approach [32] to describe these relaxation processes and ultraslow modes.

\subsection{Short-range correlation relaxation}

It follows from (65) that in equilibrium $(t \rightarrow \infty)$

$$
\left\langle\tilde{\mu}_{s}(x, t) \tilde{\mu}_{f}(x, t)\right\rangle \approx-\delta_{s} \delta_{f}
$$

and

$$
\begin{aligned}
& \delta_{h}^{2}(\infty)=\delta_{s}^{2}+\delta_{f}^{2}-2\left\langle\tilde{\mu}_{s}(x, \infty) \tilde{\mu}_{s}(x, \infty)\right\rangle \approx \\
& \approx\left(\delta_{s}+\delta_{f}\right)^{2} .
\end{aligned}
$$

In (92), (93) the thermal fluctuations of the random fields within a CD are not taken into account, as is justifiable if (see (52))

$$
2 \delta_{s} \delta_{f}>T \tilde{B} / z_{c d}
$$

i.e., if the fluctuations of the product (92) are much smaller than its average value.

The solid-fluid short-range order correlation (64) relaxes to the equilibrium value (92) if the condition (94) is fulfilled. An elementary short-range structure transformation involves not a single molecule but a group of them. The conventional name of such group is the cooperatively rearranging domain (CRR). The part of the average free energy of the CRR which varies with $\delta_{h}^{2}(t)$ is

$$
\left\langle\tilde{g}_{c r r}\right\rangle \approx \frac{z_{c r r}}{\tilde{B}} \delta_{h}^{2}(t)
$$

where $z_{c r r}$ is the number molecules in CRR.

The average free energy deviation from the equilibrium value of these molecules due to the difference

$$
\Delta \delta_{h}^{2}(t)=\delta_{h}^{2}(t)-\delta_{h}^{2}(\infty)
$$

is evidently $\sim z_{c r r} \tilde{B}^{-1} \Delta \delta_{h}^{2}$, and thus the dynamics of the short-range correlation is described by the following equation: 


$$
\begin{aligned}
& \frac{1}{2} \frac{\partial \delta_{h}^{2}(t)}{\partial t}=\frac{\partial\left\langle\delta \mu_{s}(t) \delta \mu_{f}(t)\right\rangle}{\partial t}= \\
& =-\frac{1}{2 \tau_{\alpha}} \frac{z_{c r r} \tilde{B}}{T}\left[\delta_{h}^{2}(t)-\delta_{h}^{2}(\infty)\right],
\end{aligned}
$$

where $\tau_{\alpha}$ is the CRR rearrangement time, which is the $\alpha$-relaxation time.

The Langevin term is not included in (97). It is not important under the condition (94). It is seen that $\tau_{\alpha}$ is a characteristic relaxation time of the short-range correlations.

\subsection{The long-range relaxation}

To describe the relaxation kinetics of $\xi(t)$ we assume that a fractal cluster consisting of CDs with the same value of $\tilde{h}$ appears as a result of an aggregation process of CDs in analogy with the aggregation of small species which are forming fractal structures [31]. The kinetic equation of fractal cluster formation in the simplest form is

$$
\frac{\partial \xi(\tilde{h}, t)}{\partial t}=\frac{R_{c}}{D} \Gamma_{\xi}\left(\frac{\xi(\tilde{h}, t)}{R_{c}}\right)^{1-D} \frac{\xi(\tilde{h}, \infty)-\xi(\tilde{h}, t)}{\xi(\tilde{h}, \infty)}
$$

where $\Gamma_{\xi}$ is the growth rate of the fractal. The last term on the right-hand side describes saturation of the fractal growth when the correlation length approaches its stable equilibrium value $\xi(\tilde{h}, \infty)$, determined by (58). To get an estimate of $\Gamma_{\xi}$ we note that the free energy of a CD within a fractal of correlation length $\xi(t)$, as is seen from (63), is

$$
\begin{aligned}
& \tilde{g}_{c d}\left(\tilde{h}^{2}, \xi\right)=\frac{z_{c d} R_{c}^{2}}{8 A_{0} r_{0}^{2}} \tilde{h}^{2}\left[1+4 e^{-1}\left(\frac{\xi}{R_{c}+\xi}\right)^{D-1}\right] \approx \\
& \approx \frac{z_{c d} R_{c}^{2}}{8 A_{0} r_{0}^{2}} \tilde{h}^{2}\left[1+4 e^{-1}\left(1-(D-1) \frac{R_{c}}{\xi}\right)\right] .
\end{aligned}
$$

Then

$$
\begin{aligned}
& \Gamma_{\xi} \sim \frac{R_{c}}{\tau_{\alpha}} \frac{z_{c} c(\tilde{h})}{T} \frac{\partial g_{c}}{\partial \xi} R_{c}= \\
& =(D-1) \frac{z_{c d}}{2 e A} \frac{c_{c d}(\tilde{h}) \tilde{h}^{2}}{\tau_{\alpha} T} \frac{R_{c}^{4}}{\xi^{2}} \equiv(D-1) \gamma_{0} \frac{\tilde{h}^{2}}{2 e A T} \frac{R_{c}^{4}}{\xi^{2}}
\end{aligned}
$$

In (100) $\tau_{\alpha}$ is taken as the characteristic time of CD rearrangement; $R_{c} / \tau_{\alpha}$ is the rate of the one step of the cluster growth; $T^{-1} R_{c} \partial g_{c d} / \partial \xi$ is the thermodynamic driving force of the fractal growth; the term $c_{c d}(\tilde{h})$ is introduced to take into account that just $c_{c d}(\tilde{h})$ of CD transformations result in the formation of CDs specified by the field strength $\tilde{h}$.

The solution of (98), (100) with the initial condition $\xi(\tilde{h}, 0)=0$ and $\xi(\infty)-\xi(t) \sim \xi(\infty)$ (not very close to the equilibrium value) is

$$
\xi(\tilde{h}, t)=R_{c}\left(\frac{(D-1)(D+2)}{D} \frac{\tilde{h}^{2} R_{c}^{2}}{2 e A T} \gamma_{0} t\right)^{\frac{1}{D+2}}
$$

To estimate the growth rate exponent it should be noted that for $D=1.5-2.5$ the exponent $1 /(D+2)$ varies from 0.29 to 0.22 .

In the vicinity of the $\xi(\infty)$, with $|\xi(\infty)-\xi(t)|<<\xi(\infty)$, the difference $|\xi(\infty)-\xi(t)|$ decreases exponentially, as is easy to see from (98).

The solution (100) describes the kinetics of fractals with the specified field strength $\tilde{h}$. This result has to be averaged with the distribution (52) to get an averaged description.

The relaxation time of the long-range correlation, $\tau_{\text {rel }}$, can be estimated substituting into (101) $\xi_{\text {av }}$ and $\delta_{h}^{2}$ instead of $\xi$ and $\tilde{h}^{2}$ :

$$
\tau_{\mathrm{rel}} \approx \frac{D}{(D-1)(D+2)} \frac{2 e A T}{R_{c}^{2} \delta_{h}^{2} \gamma_{0}}\left(\frac{\xi_{\mathrm{av}}}{R_{c}}\right)^{D+2} .
$$

\subsection{Ultraslow modes}

To get a dynamical equation for the Fourier components $\tilde{\alpha}_{q}$ of the long-range order parameter fluctuations, the free energy (45) has to be presented in a proper form:

$$
\begin{aligned}
& \tilde{G}_{1}=-\frac{1}{2} \int \tilde{h}(x) \tilde{a}(x) d^{3} x=-\frac{1}{2} \sum_{q} \tilde{h}_{q} \tilde{a}_{q}= \\
& =-\frac{1}{2} \sum_{q}\left(A q^{2}+\tilde{B}\right)\left|\tilde{\alpha}_{q}^{2}\right| .
\end{aligned}
$$

Then the time-dependent GL equations for $\tilde{\alpha}_{q}$ are

$$
\frac{\partial \tilde{\alpha}_{q}}{\partial t}=-\Gamma_{q} \frac{1}{T} \frac{\partial \tilde{G}_{1}}{\partial \tilde{\alpha}_{q}}=-\Gamma_{q}\left(\frac{A q^{2}+\tilde{B}}{T}\right) \tilde{\alpha}_{q} .
$$

Here the Langevin force providing the equilibrium value of $\left\langle\tilde{\alpha}_{q}^{2}\right\rangle$ is omitted because our main goal is to get the spectrum of ultraslow modes.

In an isotropic medium

$$
\Gamma_{q}=\Gamma_{0}+\gamma_{1} q^{2}+\gamma_{2} q^{4}+\ldots
$$

As follows from textbooks (e.g., [32]), the parameter $\Gamma_{0}$ in our case is equal to 0 due to conservation of $\left|\tilde{\alpha}_{q=0}^{2}\right|=\langle\tilde{\alpha}(x)\rangle^{2}=0$. Therefore 


$$
\Gamma_{q}=\gamma_{1} q^{2}+\gamma_{2} q^{4}+\ldots
$$

This equation determines the form of the ultraslow mode spectrum.

Because the CD transformations which are controlling the fractal growth are also responsible for the fractal structure rearrangements, we can put

$$
\gamma_{1} \sim \gamma_{0} R_{c}^{2} \text { and } \gamma_{2} \sim \gamma_{0} R_{c}^{4}
$$

The factors $R_{c}^{2}, R_{c}^{4}$ are included to preserve the correct dimension of $\Gamma_{q}$. It is seen from (104), (106), and (107) that the ultraslow mode spectrum has the following form:

$$
\begin{aligned}
& \omega_{u s}(q)=\left(\gamma_{1} q^{2}+\gamma_{2} q^{4}\right)\left(\frac{A q^{2}+\tilde{B}}{T}\right)= \\
& =c_{1} \tilde{B} \gamma_{0} R_{c}^{2} q^{2}+\left(\frac{c_{1} A+c_{2} \tilde{B} R_{c}^{2}}{T}\right) \gamma_{0} R_{c}^{4} q^{4}+\ldots \approx \\
& \approx c_{1} \gamma_{0} R_{c}^{2} q^{2} \frac{\tilde{B}}{T}\left(1+\frac{c_{1}+c_{2}}{c_{1}} R_{c}^{2} q^{2}\right),
\end{aligned}
$$

where $c_{1}, c_{2}$ are some $q$-independent coefficients which can be considered as constants in the critical region. The resulting expression of the ultraslow mode spectrum is obtained taking into account that $A / \tilde{B} \sim \operatorname{Tr}_{0}^{2} / \tilde{B} \approx R_{c}^{2}$.

\section{Discussion and concluding remarks}

In the theory developed, based on the concept of heterophase fluctuations, the multiplicity of the SRO, which is a basic property of the glass-forming liquids, is taken into account. In particular this property manifests itself in the developed inherent structure and in the free-energy landscape [27,28]. The free-energy landscape results in the appearance of the random-field term in the GL equations.

The relation of the present theory to the other models of supercooled liquid and to the theory of the critical fluctuations was already elucidated. To emphasize this connection it should be noted that the RFGLM developed can be reduced to the models of supercooled liquid proposed in [13-23]. For example, to get the equations of two-state model $[13,17]$ from $(26)-(30)$ we have to put $\delta_{s}^{2}=\delta_{f}^{2}=$ $=\Delta \mu_{\text {int }}=A=0$, and $k_{0}=1$.

Multiplicity of the short-range order results in polychromatic fractal heterophase structure. Polychromatic cluster structures are described, e.g., in [33] in connection with the percolation problem. If clusters have a property described by a parameter, this last one can be used as a «color» specifying a cluster. In a three-dimensional system the percolation threshold is about 0.15. Thus several interpercolating clusters can form a polychromatic structure. Along with that, any number of differently colored clusters can coexist if they have finite correlation lengths. In the heterophase liquid $\tilde{h}_{c}$ plays the role of $\mathrm{CD}$ color. It parameterizes the chromatic spectrum of CDs. The correlation lengths are finite. Thus Fischer clusters are polychromatic fractal aggregations. A specific feature of these polychromatic structures is ability of a CD to change its color.

It is worth noting that if the conditions (51), (69) are satisfied the field $\tilde{h}$ changes criticality (the mean field approximation is correct), the location of the critical point (81), and the order of the phase transition at the critical point. The order parameter jump at $T_{c}\left(\delta_{h}, P\right)$ is $\sim\left(\delta_{h} / T_{e}\right)^{1 / 3}$. Relation (81) shows that the random field lowers the critical temperature. This is one of reasons why the majority of the glass-forming liquids do not have a polymorphous liquid-liquid phase transition.

Since the mean field model (13) is isomorphic to the van der Waals theory of the critical point and (26) is a generalization of this theory taking into account the gradient term, one could ask why the random field $\tilde{h}(x)$ does not play a significant role in the critical fluctuations of gas - liquid systems. It is due to predominance of the thermal fluctuations in the gas - liquid critical region. The Ginzburg number is near 1 for the gas-liquid phase transition, i.e., $\left(a / r_{0}\right)^{6} \sim 1$, and conditions (36), (82), and (85) are not fulfilled. According to relation (36), the Ginzburg number is proportional to $k_{0}^{-2}$. Therefore within the gas - liquid critical region $k_{0}^{-2} \sim 1$, i.e., the assossiativity of the molecules is small, $\sim 1$, and the fluctuational region is rather wide. In the solid-fluid system the correlation radius far from the critical point, $r_{0}$, is approximately equal to the radius of the first coordination shell. Therefore $k_{0}$ is $\sim 10$ in this case and, accordingly to (36), the mean field theory has a wide region of applicability even if $\tilde{h}(x)$ is neglected.

The random-field-driven LRC qualitatively differs from the conventional critical LRC. The former LRC possess the properties 3)-5) of the Fischer cluster (see Introduction), while the latter LRC cannot have them. In $[18,19]$ it was attempted to identify the Fischer cluster as critical fluctuations, assuming that the critical temperature is below $T_{g}$. The analysis performed in [12] shows that the theory proposed in $[18,19]$ for the LRC observed by Fischer et al. in ortho-terphenil does not fit the ex- 
perimental data even if the features 3)-5) of Fischer's cluster are ignored.

Glass inherits the structural features of supercooled liquid. For this reason short-range and longrange correlations of liquid are also present in the glassy state. Because the annealing time needed for the Fischer cluster formation is rather long compared to $\tau_{\alpha}$, the structure and properties of the glass are sensitive to the thermal history of the liquid. The correlations and topology of SRO in the glassy state play a rather important role in phase transformations taking place in glasses. For example, ordering and relaxation of the spin system of a magnetic glass are extremely sensitive to the structural and compositional correlations [34]. The structural heterogeneities play the role of a «frozen» random field in this case. Another example is the polymorphous phase transitions in glassy state. This phenomenon is observed in some glasses (see $[35,36])$. It is revealed also in amorphous carbon using computer simulations [37]. Because glass is a nonergodic system, a phase transformation is possible due to changes of SRO resulting in a longrange ordering. The structural heterogeneities cause an inhomogeneous distribution of internal stresses due to thermoelastic effects. Therefore the transformations of the SRO are necessarily inhomogeneous and depend on the distribution and correlation properties of the internal stresses and compositional ordering.

A topological phase transition connected with the formation of a percolating cluster possessing some SRO stipulates a thermodynamic phase transition due to the singular behavior of the thermodynamic coefficients, e.g., the compressibility or thermal expansion. Therefore polymorphous phase transitions in the glassy state have to be sensitive to structural correlations that depend on the thermal history of the liquid.

Recently anomalous behavior of the thermal expansion coefficient $\lambda(T)$ of pure and inert-gas doped fullerite at $T<20 \mathrm{~K}$ was observed [38,39]. Fullerite $\mathrm{C}_{60}$ is a fcc cubic crystal. Due to the fivefold symmetry of the $\mathrm{C}_{60}$ molecules, long-range orientational order is impossible in fullerite. For this reason fullerite is an orientational glass below $T_{g} \cong$ $\cong 90 \mathrm{~K}$. Impurities distort the crystalline structure and disturb the intermolecular interactions in the fullerite. The thermal expansion coefficient depends considerably on the concentration and kind of inert gas impurities. It is remarkable that hysteresis of $\lambda(T)$ was observed at $T<20 \mathrm{~K}$ in krypton-doped fullerite. It is attributed to a phase transition connected with changes of the short-range orientational order of the $\mathrm{C}_{60}$ molecules. One can expect that the observed hysteresis phenomenon depends considerably on the concentration of krypton and on the thermal history of samples at $T>T_{g}$ due to changes of the short-range and medium-range orientational order in the orientational liquid state.

Many fruitful discussions with E. W. Fischer are cordially acknowledged.

This work was supported in part by the Science and Technology Center of Ukraine, project N655.

1. H. Silescu, J. Non-Cryst. Solids 243, 81 (1999).

2. E. W. Fischer, Ch. Becker, I.-U. Hagenah, and G. Meier, Prog. Colloid Polym. Sci. 80, 198 (1989).

3. E. W. Fischer, G. Meier, T. Rabenau, A. Patkowski, W. Steffen, and W. Thonnes, J. Non-Cryst. Solids 131-133, 134 (1991).

4. E. W. Fischer, Physica A201, 183 (1993).

5. T. Kanaya, A. Patkowski, E. W. Fischer, J. Seils, H. Glaser, and K. Kaji, Acta Polymer. 45, 137 (1994).

6. A. Patkowski, Th. Thurn-Albrecht, E. Banachowicz, W. Steffen, T. Narayan, and E. W. Fischer, Phys. Rev. E61, 6909 (2000).

7. A. Patkowski, E. W. Fischer, W. Steffen, H. Glaeser, M. Baumann, T. Ruths, and G. Meier, Phys. Rev. E63, 061503 (2001).

8. A. Patkowski, H. Glaeser, T. Kanaya, and E. W. Fischer, Phys. Rev. E64, 031503 (2001).

9. E. W. Fischer, A. Bakai, A. Patkowski,W. Steffen, and L. Reinhardt, J. Non-Cryst. Solids (2002) (in press).

10. K. Kawasaki, Physica A217, 124 (1995).

11. H. Tanaka, J. Chem. Phys. 111, 3163; 3175 (1999); Phys. Rev. E62, 6968 (2000).

12. A. S. Bakai, J. Non-Cryst. Solids 307-310, 623 (2002).

13. P. B. Macedo, W. Capps, and T. A. Litovitz, J. Chem. Phys. 44, 3357 (1966).

14. I. L. Apteker and E. G. Ponyatovsky, Dokl. Acad. Nauk SSSR 173, 851 (1967); E. G. Ponyativsky and O. I. Barkalov, Mat. Sci. Rep. 8, 147 (1992).

15. E. Rapoport, J. Chem. Phys. 46, 2891 (1967).

16. C. A. Angell and K. I. Rao, J. Chem. Phys. 57, 470 (1972).

17. M. H. Cohen and G. S. Grest, Phys. Rev. B20, 1077 (1979); ibid. B26, 6313 (1982).

18. I. T. Bendler and M. F. Shlessinger, J. Stat. Phys. 53, 531 (1988).

19. I. T. Bendler and M. F. Shlessinger, J. Chem. Phys. 96, 3970 (1992).

20. A. S. Bakai, in: Topics in Appl. Phys., v. 72, Glassy Metals III, H. Beck and H.-J. Guentherodt (eds.), Springer, Heidelberg (1994), p. 209.

21. A. S. Bakai, Fiz. Nizk. Temp. 20, 469 (1994) [Low Temp. Phys. 20, 373 (1994)]. 
22. A. S. Bakai, Fiz. Nizk. Temp. 22, 956 (1996); ibid. 24, 27 (1998) [Low Temp. Phys. 22, 733 (1996); ibid. 24, 20 (1998)].

23. E. W. Fischer and A. S. Bakai, Slow Dynamics in Complex Systems, AIP Conf. Proc. 469, M. Tokuyama and I. Oppenheim (eds.) (1999), p. 325.

24. L. D. Landau and E. M. Lifshits, Statistical Physics: Course of Theoretical Physics, vol. 5, Nauka, Moscow (1976) (in Russian).

25. A. S. Bakai, Cond. Mat. Phys. 3, 675 (2000).

26. H. Sher and R. Zallen, J. Chem. Phys. 53, 3759 (1970); R. Zallen and H. Sher, Phys. Rev. B4, 4471 (1971).

27. F. H. Stillinger and T. A. Weber, Phys. Rev. A28, 2408 (1983).

28. S. Sastry, P. G. Debenedetty, and F. H. Stillinger, Nature (London) 394, 554 (1998).

29. Vik. S. Dotsenko, Uspekhi Fiz. Nauk 165, 481 (1995).

30. Spin Glasses and Random Fields, A. P. Young (ed.), World Sci. Publ. Co. Ltd., Singapore (1998).

31. J. Feder, Fractals, Plenum Press, New York (1988).

32. S. Ma, Modern Theory of Critical Phenomena, W. A. Benjamin, Inc., (1976).
33. R. Zallen, The Physics of Amorphous Solids, John Willey \& Sons, New York (1983).

34. A. S. Bakai, Fiz. Nizk. Temp. 28, 584 (2002) [Low Temp. Phys. 28, 415 (2002)].

35. C. A. Angell, Science 267, 1924 (1994).

36. P. H. Pool, T. Grande, F. Sciortino, H. E. Stanley, and C. A. Angell, Coputational Mater. Science 4, 373 (1995).

37. A. S. Bakai, M. P. Fateev, and Yu. A. Turkin, in: Nanostructured Carbon for Advanced Applications, NATO ASI series, G. Benedeck et al. (eds.), Kluwer Ac. Publishers (2001), p. 185; Preprint CondMat/0108518 (2001).

38. A. N. Alexandrovskii, V. G. Gavrilko, V. B. Essel'son, V. G. Manzhelii, B. G. Udovichenko, and V. P. Maletskiy, Fiz. Nizk. Temp. 27, 1401 (2001) [Low Temp. Phys. 27, 1033 (2001)].

39. A. N. Aleksandrovskii, A. V. Dolbin, V. B. Esel'son, G. Gadd, V. G Gavrilko, V. G. Manzhelii, B. Sundqvist, and B. G. Udovidchenko (to be published). 